\title{
Reflectivity and Microwave Absorption in Crystals with Alternating Intrinsic Josephson Junctions
}

\author{
Ch. Helm ${ }^{1,2}$, L.N. Bulaevskii ${ }^{1,3}$, E.M. Chudnovsky ${ }^{3}$, and M.P. Maley ${ }^{1}$ \\ ${ }^{1}$ Los Alamos National Laboratory, Los Alamos, NM 87545 \\ ${ }^{2}$ Institut für Theoretische Physik, ETH Hönggerberg, 8093 Zürich, Switzerland \\ ${ }^{3}$ Physics Department, CUNY Lehman College, Bronx, NY 10468-1589
}

(October 28, 2018)

\begin{abstract}
We compute the frequency and magnetic field dependencies of the reflectivity $R(\omega)$ in layered superconductors with two alternating intrinsic Josephson junctions with different critical current densities and quasiparticle conductivities for the electric field polarized along the $c$-axis. The parameter $\alpha$ describing the electronic compressibility of the layers and the charge coupling of neighboring junctions was extracted for the $\mathrm{SmLa}_{1-x} \mathrm{Sr}_{x} \mathrm{CuO}_{4-\delta}$ superconductor from two independent optical measurements, the fit of the loss function $L(\omega)$ at zero magnetic field and the magnetic field dependence of the peak positions in $L(\omega)$. The experiments are consistent with a free electron value for $\alpha$ near the Josephson plasma frequencies.
\end{abstract}

The Josephson plasma resonance (JPR) observed in the microwave absorption [1], and in optical reflection [20] and transmission measurements [6]7], has proven to be a powerful method to study the properties of highly anisotropic layered superconductors, such as vortex phases [1,8]. In particular, the spatial dispersion of the JPR, $\omega_{p}\left(\mathbf{k}, k_{z}\right)$, parallel $(\mathbf{k})$ and perpendicular $\left(k_{z}\right)$ of the superconducting layers reflects the inductive coupling of junctions due to intralayer currents and the charge coupling due to the variations of the electrochemical potential on the layers, respectively. Determining the latter is essential for understanding the coupled dynamics of the stack of intrinsic Josephson junctions in cuprate superconductors, e.g. with respect to coherence in $\mathrm{THz}$ emission [9.10]. From a fundamental point of view, it contains unique information about the electronic structure of the superconducting $\mathrm{CuO}_{2}$-layers, namely their compressibility, which is hard to obtain otherwise. Beyond this, from the damping of the JPR the $c$-axis quasiparticle (QP) conductivity at the JPR frequency can be extracted.

The parameter $\alpha$ characterizing the $c$-axis dispersion of the JPR in crystals with identical junctions is difficult to observe in bulk microwave, transport [10] or optical experiments, because mainly modes with small $k_{z}$ are excited, although for grazing incidence the JPR peak amplitude can depend on $\alpha$ [11]. The latter is irrelevant for the experiments in $[2$ 5], which are performed with incidence parallel to the layers, which will be studied here. In the following we will show, how $\alpha$ can be extracted unambiguously for layered superconductors with a superstructure in $c$-direction from two independent measurements of the loss function and the magnetic field dependence of the plasma frequencies.

Recently, these experiments have been performed on $\mathrm{SmLa}_{1-x} \mathrm{Sr}_{x} \mathrm{CuO}_{4-\delta}$, where magnetic $\mathrm{Sm}_{2} \mathrm{O}_{2}$ and nonmagnetic $\mathrm{La}_{2-x} \mathrm{Sr}_{x} \mathrm{O}_{2-\delta}$ layers alternate in the barriers between the $\mathrm{CuO}_{2}$-layers [3 6]. In Refs. [3 5 the reflectivity $R$ and transmission of the electromagnetic wave propagating along the layers and with the electric field along the $c$-axis (cf. Fig. 1a) were used to extract the effective dielectric function $\epsilon_{\text {eff }}(\omega)$ with the help of the Fresnel formulas, e.g. $R(\omega)=[1-$ $\left.\sqrt{\epsilon_{\mathrm{eff}}(\omega)}\right] /\left[1+\sqrt{\epsilon_{\mathrm{eff}}(\omega)}\right]$. The authors reported two peaks with quite similar widths in the loss function, $L(\omega)=\operatorname{Im}\left[-1 / \epsilon_{\mathrm{eff}}(\omega)\right]$, and a quite large ratio of the peak intensities $L\left(\omega_{1}\right) / L\left(\omega_{2}\right)$ between 10 and 20 , see Fig. 2. In a two-junction model with two different plasma frequencies, but identical quasiparticle conductivities and no charge coupling the peak amplitudes in the loss function are quite similar, see Fig. 2 at $\alpha=0$. This disagreement with the experimental data cannot be explained by a strong frequency dependence of the conductivities, as the widths of the two peaks are too similar. Instead, it was argued in [5] 12] that the $c$-axis coupling plays a crucial role for the peak intensities.
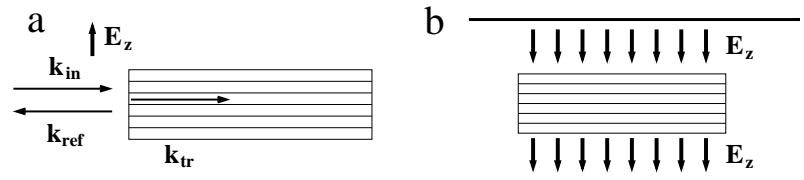

FIG. 1. Geometry for measuring (a) the reflectivity with parallel incidence and (b) the microwave absorption in a cavity with the electric field polarized along the $z$-axis.

However, in the derivation of $\epsilon_{\mathrm{eff}}$ in Ref. [12] dissipation was not introduced in the Maxwell equations, but arbitrarily in the final expression for $\epsilon_{\text {eff }}$ calculated without dissipation. In this Letter we correct these results accounting for different tunneling conductivities in the junctions in accordance with their different critical current densities. We compare the theoretical $L(\omega)$ with the experimental data in [3,13] and extract at the JPR peaks the parameter $\alpha \approx 0.4$, which is the free electron value. Further, we show that the microwave absorption 
in the spatially uniform AC electric field applied along the $c$-axis (see Fig. 1b) is also determined by $L(\omega)$.

Recently, Pimenov et al. [6] measured the dependence of the plasma frequencies on the magnetic field $\mathbf{B} \| c$. We show that the field dependence of the peak positions in $L(\omega)$ alone allows us to extract $\alpha \approx 0.4$, which is an independent confirmation of the fit of $L(\omega)$ for $B=0$.

We also show that the QP conductivities extracted at two different frequencies do not show the $\omega$-dependence as anticipated for gapless $d$-wave pairing. We argue that the properties of the Cooper pair tunneling via the magnetic Sm ions may overshadow the $\omega$-dependence of the QP conductivity.

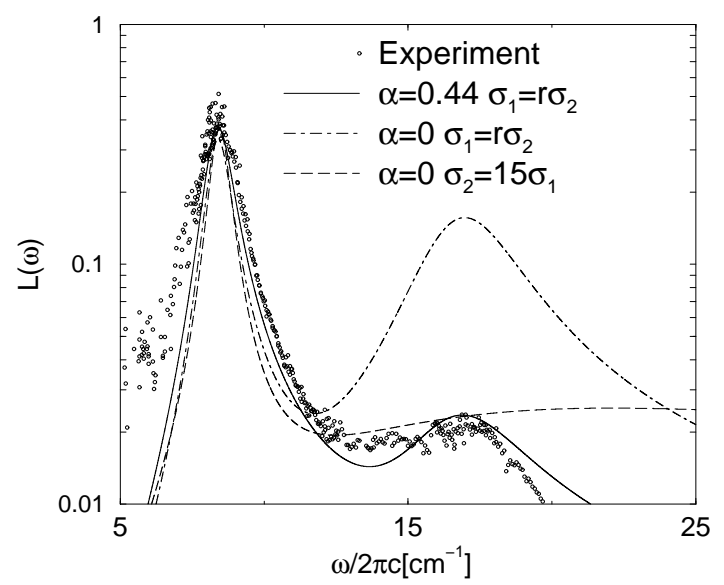

FIG. 2. Experimental data [13] together with the calculated loss function $L(\omega)$ (solid: $\alpha=0.44, \tilde{\sigma}_{1}=r \tilde{\sigma}_{2}=0.12$, $r=0.38, \omega_{0,1} / c=7.2 \mathrm{~cm}^{-1}, \epsilon_{0}=18$; dot-dashed: $\alpha=0$, $\tilde{\sigma}_{1}=r \tilde{\sigma}_{2}=0.12, r=0.24, \omega_{0,1} / c=8.3 \mathrm{~cm}^{-1}, \epsilon_{0}=13$; dashed: $\alpha=0, \tilde{\sigma}_{2}=15 \tilde{\sigma}_{1}=0.2, r=0.24, \omega_{0,1} / c=8 \mathrm{~cm}^{-1}$, $\left.\epsilon_{0}=15\right)$.

We consider the crystal with two alternating Josephson junctions characterized by different critical current densities, $J_{l}$, and the $c$-axis tunneling conductivities, $\sigma_{l}$, due to different tunneling matrix elements. We assume that all other parameters of the junctions are identical, as their distinction would lead to negligible corrections in the following. Without the charging effect the $c$-axis bare plasma frequencies $\omega_{0, l}$ are related to $J_{l}$ as $\omega_{0, l}^{2}=8 \pi^{2} c s J_{l} / \epsilon_{0} \Phi_{0}$, where $\Phi_{0}$ is the flux quantum, $\epsilon_{0}$ is the high frequency $c$-axis dielectric constant and $s$ is the interlayer distance. We neglect nonequilibrium effects [10,14 in the distribution function of the quasiparticles assuming that the plasma frequencies are well below the charge imbalance and energy relaxation rates.

To find the reflectivity $R(\omega)$ in parallel incidence and the microwave absorption, we use the Maxwell equations inside the crystals accounting for supercurrents inside the 2 D layers at $z=m s$ and interlayer Josephson and quasiparticle currents determined by the difference of the electrochemical potentials in neighboring layers: $c \frac{\partial B_{y}}{\partial z}=i \epsilon_{a 0} \omega\left[E_{x}-\frac{\omega_{a 0}^{2}}{\omega^{2}} \sum_{m=1}^{N} E_{x} s \delta(z-m s)\right]$,

$\frac{\partial E_{x}}{\partial z}-i k_{x} E_{z}=i \frac{\omega}{c} B_{y}, \quad E_{z, m, m+1}=\int_{m s}^{(m+1) s} E_{z} \frac{d z}{s}$

$c k_{x} B_{y}=-\omega \epsilon_{0}\left[E_{z}-\sum_{m=1}^{N} A_{m} f_{m, m+1}(z)\right]$,

$\frac{\tilde{\omega}_{l}^{2} e s}{\omega_{0, l}^{2}} A_{m}=V_{m, m+1}=e s E_{z, m, m+1}+\mu_{m+1}-\mu_{m}$.

Here $\mu_{m}$ is the chemical potential in the layer $m, V_{m, m+1}$ is the difference of the electrochemical potentials, $\omega_{a 0}=$ $c / \lambda_{a b} \sqrt{\epsilon_{a 0}}$ is the in-plane plasma frequency and $\epsilon_{a 0}$ is the high frequency in-plane dielectric constant. The function $f$ is defined as $f_{m, m+1}(z)=1$ at $m s<z<(m+1) s$ and zero outside this interval. To obtain Eq. (3) for small amplitude oscillations we expressed the supercurrent density $J_{m, m+1}^{(s)}=J_{l} \sin \varphi_{m, m+1} \approx J_{l} \varphi_{m, m+1}$ via the phase difference $\varphi_{m, m+1}=2 i V_{m, m+1} / \hbar \omega$. Further, $\tilde{\omega}_{l}^{2}=\omega^{2}(1-$ $\left.i 4 \pi \sigma_{l} \omega / \omega_{0, l}^{2} \epsilon_{0}\right)^{-1}$ takes into account the dissipation due to QP tunneling currents, $J_{m, m+1}^{(q p)}=\sigma_{l} V_{m, m+1} / e s$, which are depend on the different conductivities $\sigma_{l}$ in the junctions $l=1,2$ and the difference $V_{m, m+1}$ of the electrochemical potentials. We express the difference of the chemical potentials $\mu_{m}$ via the difference of the $2 \mathrm{D}$ charge densities, $\rho_{m}$, as $\mu_{m}-\mu_{m+1}=\left(4 \pi s \alpha / \epsilon_{0}\right)\left(\rho_{m}-\rho_{m+1}\right)$, where the parameter $\alpha=\left(\epsilon_{0} / 4 \pi e s\right)(\partial \mu / \partial \rho)$ characterizes the interlayer coupling. In the model of $2 \mathrm{D}$ free electrons we find $\partial \mu / \partial \rho=\pi \hbar^{2} /\left(\mathrm{em}^{*}\right)$. For an effective mass $m^{*} \approx 1-2 m_{e}$, as expected from ARPES [15], $s=6.3 \AA$ and $\epsilon_{0}=20$ we estimate the order of $\alpha$ as $\approx 0.2-0.4$.

The solution of the Eqs. (11)-(侜) is

$$
\begin{aligned}
& B_{y}(z)=\frac{\epsilon_{0} \omega}{c k_{x}}\left[c_{m} \exp (i g z)+d_{m} \exp (-i g z)\right]-a \frac{c k_{x}}{\omega} A_{m} \\
& E_{x}(z)=\frac{\epsilon_{0} \omega}{\left(\epsilon_{a 0} a\right)^{1 / 2} c k_{x}}\left[c_{m} \exp (i g z)-d_{m} \exp (-i g z)\right] \\
& E_{z}(z)=-\left[c_{m} \exp (i g z)+d_{m} \exp (-i g z)\right]+a A_{m}
\end{aligned}
$$

where now $0 \leq z \leq s$ in each junction, $g^{2}=\omega^{2} \epsilon_{a 0} /\left(c^{2} a\right)$ and $a^{-1}=1-c^{2} k_{x}^{2} / \epsilon_{0} \omega^{2}$. The Maxwell boundary conditions for $B_{y}$ and $E_{x}$ across the layers lead to a set of equations for $c_{m}, d_{m}$ and $A_{m}$, which we solve using the small parameters $b=g s / 2 \sim s / \lambda_{c} \ll 1$ and $\beta=s^{2} / 2 \lambda_{a b}^{2} a \ll 1$. In order to find the reflectivity for parallel incidence, cf. Fig. 1a, we consider the case $s k_{z} \ll b \lesssim \beta$, which is fulfilled for incident angles $\theta \ll 1$, and obtain the dispersion relation $k_{x}(\omega)$ for waves propagating inside the crystal (for details see [16):

$$
\begin{aligned}
& \frac{c^{2} k_{x}^{2}}{\omega^{2} \epsilon_{0}}=\frac{\epsilon_{\mathrm{eff}}(w)}{\epsilon_{0}}=\frac{r\left(w-v_{1}\right)\left(w-v_{2}\right)+i S}{r w^{2}-(1+r)(2 \alpha+1 / 2) w+i S_{1}}, \\
& v_{1,2}=(1+r)(1+2 \alpha)(1 \mp \sqrt{1-p}) / 2 r \\
& p=\frac{4 r(1+4 \alpha)}{(1+r)^{2}(1+2 \alpha)^{2}}
\end{aligned}
$$


$S_{1}=w^{3 / 2} r(2 \alpha+1 / 2)\left(\tilde{\sigma}_{1}+\tilde{\sigma}_{2}\right)$,

$S=w^{1 / 2}\left[(2 \alpha+1) r w\left(\tilde{\sigma}_{1}+\tilde{\sigma}_{2}\right)-(1+4 \alpha)\left(\tilde{\sigma}_{1}+\tilde{\sigma}_{2} r\right)\right]$,

where $v_{l}=\omega_{l}^{2} / \omega_{0,1}^{2}, \omega_{l}$ are the JPR frequencies, $w=$ $\omega^{2} / \omega_{0,1}^{2}, \tilde{\sigma}_{l}=4 \pi \sigma_{l} / \epsilon_{0} \omega_{0,1}$ and $r=\omega_{0,1}^{2} / \omega_{0,2}^{2}<1$.

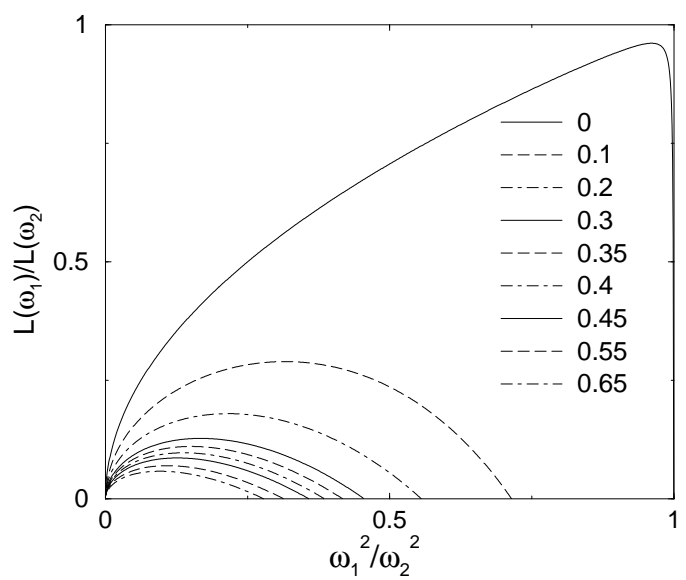

FIG. 3. Ratio of the peak amplitudes $L\left(\omega_{1}\right) / L\left(\omega_{2}\right)$ in the loss function depending on the squared ratio $\omega_{1}^{2} / \omega_{2}^{2}$ of the JPR frequencies for different $\alpha=0, \ldots, 0.65$ (top to bottom plot) at $\tilde{\sigma}_{1}=r \tilde{\sigma}_{2}$.

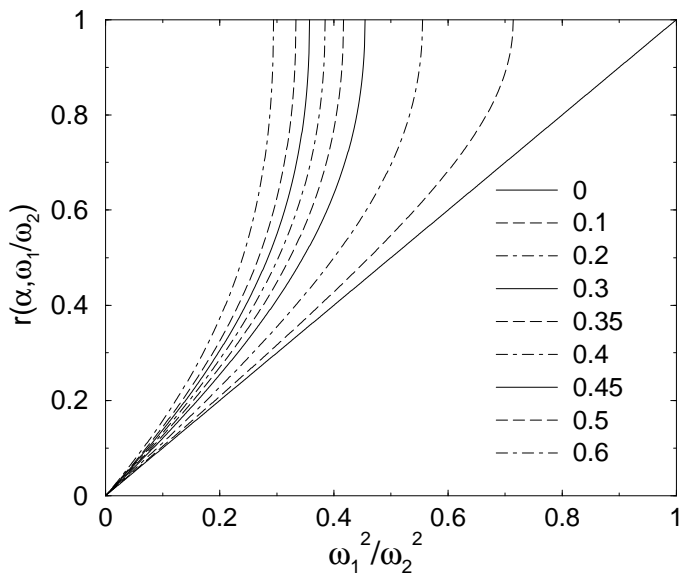

FIG. 4. Ratio $r=\omega_{0,1}^{2} / \omega_{0,2}^{2}$ of the squared bare plasma frequencies depending of the squared ratio $\omega_{1}^{2} / \omega_{2}^{2}$ of the JPR resonances in $L(\omega)$ for different $\alpha=0, . ., 0.6$ (from below).

The reflectivity coefficient is given by the usual Fresnel expression $R=\left(1-c k_{x} / \omega\right) /\left(1+c k_{x} / \omega\right)$. Note that for nonzero $\beta$ we obtain in principle two propagating modes inside the crystal, but the second mode gives a negligible contribution near the JPR peaks at $\beta \ll 1$, see 16. The loss function is

$$
\begin{aligned}
L(w)= & \operatorname{Im}\left[-1 / \epsilon_{\mathrm{eff}}(\omega)\right]=\left(w^{3 / 2} / 2 \epsilon_{0}\right) \times \\
& \frac{\tilde{\sigma}_{1}(w r-4 \alpha-1)^{2}+\tilde{\sigma}_{2} r^{2}(w-4 \alpha-1)^{2}}{\left[r\left(w-v_{1}\right)\left(w-v_{2}\right)\right]^{2}+S^{2}}
\end{aligned}
$$

and shows resonances at the two transverse plasma bands, while the peak in $\operatorname{Im}\left[\epsilon_{\text {eff }}(\omega)\right]$ is at $v_{T}=(1+$ $1 / r)(2 \alpha+1 / 2)$.

In $s$-wave superconductors with nonmagnetic ions in the barrier between the layers both the QP conductivities $\sigma_{l}$ and the critical current densities $J_{l}$ vary with $l$ only due to their proportionality to the squared matrix element for the interlayer tunneling in agreement with the doping dependence $\sigma_{c}(x) \propto \omega_{p}^{2}(x)$ found for $\mathrm{La}_{2-x} \mathrm{Sr}_{x} \mathrm{CuO}_{4}$ [17]. We take the same relation, $\sigma_{1}\left(\omega_{1}\right) / \sigma_{2}\left(\omega_{2}\right)=r$ for $\mathrm{SmLa}_{1-x} \mathrm{Sr}_{x} \mathrm{CuO}_{4-\delta}$, ignoring the frequency dependence of $\sigma_{l}$ due to the $d$-wave pairing [18], which decreases $\sigma_{1}\left(\omega_{1}\right) / \sigma_{2}\left(\omega_{2}\right)$ below $r$. On the other hand, we also neglect that due to the magnetism of the $\mathrm{Sm}$ ions the critical current density $J_{1} \propto \omega_{0,1}^{2}$ is suppressed, while $\sigma_{l}$ is not affected, which suggests $\sigma_{1} / \sigma_{2}>r$ without $d$-wave pairing [19]. The fact that our assumption $\sigma_{1}=r \sigma_{2}$ turns out to be consistent with the experimental data suggests that both effects compensate each other.

In Fig. 3 we present the dependence of the ratio of the peak amplitudes in the loss function $L(\omega)$, i.e. $L\left(\omega_{1}\right) / L\left(\omega_{2}\right)$, vs. the ratio $\omega_{1}^{2} / \omega_{2}^{2}$ for different values of $\alpha$. Hence, this figure allows us to obtain the parameter $\alpha$ from the positions $\omega_{1,2}$ of the JPR peaks and their ratio of amplitudes. Fig. 1 allows us to find the ratio of the squared bare frequencies, $r=\omega_{0,1}^{2} / \omega_{0,2}^{2}$ for a ratio $\omega_{1} / \omega_{2}$ when the parameter $\alpha$ is obtained from Fig. 3 . The comparison of $L(\omega)$ in Fig. 2 with the experimental data from Ref. [13] gives the best fit for $\tilde{\sigma}_{1}=r \tilde{\sigma}_{2}=0.12$ and $\epsilon_{0}=18, \alpha=0.44$, which is of the same order as the theoretical estimate for free electrons, cf. Tab. I for a fit of [3,5, 6, 13]. In the case $\alpha=0$ a high ratio $\tilde{\sigma}_{2} / \tilde{\sigma}_{1} \approx 15$ of the conductivities is necessary to reproduce the ratio of the amplitudes, which fails to describe the shape of the resonance at $\omega_{2}$ correctly.

The loss function $L(\omega)$ also determines the microwave absorption of a crystal in a capacitor, which induces a uniform $\left(s k_{z} \rightarrow 0, k_{x}=0\right)$ AC electric field $2 \mathcal{E} \cos (\omega t)$ above and below the crystal, see Fig. 1b. The crystal excitations are longitudinal in this case, i.e. $E_{x}=B_{y}=0$ in Eqs. (11)-(任). These equations together with the Poisson equations near the top and bottom layers determine for large $N$ the microwave absorption as

$$
\mathcal{P}(\omega)=N^{-1} \sum_{m=1}^{N} \sigma_{l}\left|V_{m, m+1} / e s\right|^{2}=\frac{\omega}{4 \pi} L(\omega) \mathcal{E}^{2} .
$$

Next we consider the dependence of the JPR peaks in $L(\omega)$ on the $c$-axis magnetic field $B$, which allows one to estimate the parameter $\alpha$, without relying on the absolute amplitude of the spectra. For Josephson junctions $B$ suppresses the critical current densities and the bare plasma frequencies, $\omega_{0, l}(B)$, and also broadens the resonance peaks due to the formation of pancake vortices randomly misaligned along the $c$-axis $[8]$. From Eq. (7) we see that the ratio of the resonance frequencies, 


$$
\omega_{1}^{2} / \omega_{2}^{2}=(1-\sqrt{1-p}) /(1+\sqrt{1-p}),
$$

depends only on the quantity $p$ given by Eq. (8), which varies with $B$ only via the function $r(B)=$ $\omega_{0,1}^{2}(B) / \omega_{0,2}^{2}(B)$. When the functional form of $\omega_{0, l}(B)$ is known theoretically and $p(B)$ is obtained from the measurement of $\omega_{1} / \omega_{2}$ via Eq. (12), one can fit unknown parameters in $\omega_{0, l}(B)$ and obtain $\alpha$. It is pointed out that extracting $\alpha$ from $L(\omega, B)$ by fitting all parameters without further theoretical input is difficult due to the dependence of the effective $c$-axis conductivity on $B$ [8] .

The dependence $\omega_{0, l}(B)$ in the decoupled vortex liquid [8] is known for crystals with equivalent junctions and can be used for alternating junctions as well, provided that the vortices in different layers are decoupled. This is suggested by $\hbar \omega_{0, l} \ll k T_{c}$ in $\mathrm{SmLa}_{0.8} \mathrm{Sr}_{0.2} \mathrm{CuO}_{4-\delta}$ (cf. Tab. I) indicating Josephson coupling of the layers. Thus, for the vortex liquid phase with decoupled pancakes in high magnetic fields $B \gg B_{J}=\Phi_{0} \lambda_{a b}^{2} /\left(\lambda_{c} s\right)^{2} \sim 1 \mathrm{~T}$ the dependence of the plasma frequencies on $B$ is [ 8$]$

$$
\omega_{0, l}^{2}(B) \approx \omega_{0, l}^{4}(0) \frac{\epsilon_{0} \Phi_{0}^{3}}{32 B \pi^{3} c^{2} s T}
$$

This gives $r\left(B \gg B_{J}\right)=r^{2}(B=0)$. Hence, we find

$$
p\left(B \gg B_{J}\right)=\frac{4 r^{2}(0)(1+4 \alpha)}{\left[1+r^{2}(0)\right]^{2}(1+2 \alpha)^{2}},
$$

while for $p(0)$ we use Eq. (8) with $r=r(0)$.

Consequently, the parameters $\alpha$ and $r(0)$ can be found analytically without fitting from $p(0)$ and $p\left(B \gg B_{J}\right)$ obtained from JPR frequency measurements using the data of Ref. [6]. The dependence $\omega_{1}^{2}, \omega_{2}^{2} \propto 1 / B$ at fields above $B_{J} \approx 1 \mathrm{~T}$ shows that in this field range the decoupled pancake liquid is present. We use the reported field dependence of the peak frequency in $\operatorname{Im}\left[\epsilon_{\text {eff }}(\omega)\right]$ for $\omega_{2}(B)$, as the authors noted that they are quite close and the difference will finally turn out to be $\approx 10 \%$. Then we obtain (for $T \ll T_{c}$ ) $\omega_{1}^{2} / \omega_{2}^{2} \approx 0.31$ at $B=0$ and 0.21 at $B>B_{J}$ or $p(0) \approx 0.73$ and $p\left(B \gg B_{J}\right) \approx 0.57$ respectively. From Eqs. (8) and (14) we obtain $r(0) \approx 0.55, \alpha \approx 0.4$ and the bare frequencies are $\omega_{0,1} / c=6.6 \mathrm{~cm}^{-1}$ and $\omega_{0,2} / c=8.9$ $\mathrm{cm}^{-1}$, cf. Tab. I. This estimate gives the parameters $\alpha$ and $r(0)$ similar to those obtained above from the fit of $L(\omega)$ at $B=0$ and confirms independently the relevance of the $c$-axis coupling $\alpha$ and our assumption $\tilde{\sigma}_{1} \approx r \tilde{\sigma}_{2}$.

\begin{tabular}{c|c|c|c|c|c|c}
\hline \hline$x$ & $T_{c}$ & $\omega_{0,1} / c$ & $r$ & $\sigma_{1}\left[(\Omega \mathrm{m})^{-1}\right]$ & $\alpha$ & Ref. \\
\hline 0.15 & $23 \mathrm{~K}$ & $10.9 \mathrm{~cm}^{-1}$ & 0.42 & 10 & 0.36 & {$[3]$} \\
0.15 & $30 \mathrm{~K}$ & $7.2 \mathrm{~cm}^{-1}$ & 0.38 & 4.3 & 0.44 & {$[13]$} \\
0.2 & $17 \mathrm{~K}$ & $6.6 \mathrm{~cm}^{-1}$ & 0.55 & 3.9 & 0.40 & {$[5,6]$} \\
\hline \hline
\end{tabular}

TABLE I. Extracted parameters for $\mathrm{SmLa}_{1-x} \mathrm{Sr}_{x} \mathrm{CuO}_{4-\delta}$
In conclusion, we calculated the effective dielectric function $\epsilon_{\text {eff }}$, Eq. (6), for alternating junctions with charge coupling $\alpha$ and frequency independent QP conductivities $\sigma_{l} \propto \omega_{0, l}^{2}$. This allows to describe satisfactory the optical properties of the $\mathrm{SmLa}_{0.8} \mathrm{Sr}_{0.2} \mathrm{CuO}_{4-\delta}$ superconductor near the plasma frequencies. The parameter $\alpha \approx 0.4$ was extracted independently from the magnetic field dependence of the positions of the JPR peaks $\omega_{1,2}(B)$ and from the fit of the loss function $L(\omega)$ at $B=0$. Its value is expected to be universal in the cuprates and corresponds to the free electron value of the electronic compressibility, which can differ from the renormalized one particle density of states both for Fermi and non Fermi liquids [20]. We also show that the extracted conductivities $\sigma_{l}$ differ according to the different tunneling matrix elements, $\sigma_{1} / \sigma_{2} \approx \omega_{0,1}^{2} / \omega_{0,2}^{2}$. This relation is anticipated for tunneling between $s$-wave superconductors via nonmagnetic ions. Its fulfillment in $\mathrm{SmLa}_{0.8} \mathrm{Sr}_{0.2} \mathrm{CuO}_{4-\delta}$ might indicate that the gapless $d$ wave pairing in the layers reduces $\sigma_{1}\left(\omega_{1}\right) / \sigma_{2}\left(\omega_{2}\right)$ in a similar way as the magnetism of the $\mathrm{Sm}$ ions decreases $\omega_{0,1}^{2} / \omega_{0,2}^{2}$. This could be studied further by measuring the gap in the QP spectrum in the $I-V$ curve as in Ref. [18].

The authors thank M. Graf and W. Zwerger for useful discussions. The work was supported by the U.S. DOE (in Lehman College through Grant No. DE-FG0293ER45487) and the NRCC of the Swiss NSF.

[1] Y. Matsuda, et al., Phys. Rev. B 55 R8685 (1997); T. Shibauchi, et al., Phys. Rev. B 55 R11977 (1997).

[2] D. Dulić, et al., Phys. Rev. B 60, R15051 (1999).

[3] H. Shibata, Phys. Rev. Lett. 86, 2122 (2001).

[4] T. Kakeshita, et al., Phys. Rev. Lett. 86, 4140 (2001).

[5] D. Dulić, et al., Phys. Rev. Lett. 86,4144 (2001).

[6] A. Pimenov, et al., Phys. Rev. Lett. 87,17 7003 (2001).

[7] V.K. Thorsmølle, et al., Opt. Lett. 26, 1292 (2001).

[8] A.E. Koshelev, et al., Phys. Rev. B 62, 14403 (2000).

[9] M. Tachiki, et al., Phys. Rev. B 50, 7065 (1994).

[10] Ch. Helm, et al., Physica C 362,43 (2001); D. Ryndyk, et al., J. Phys.: Cond. Mat. 14, 815 (2002); Ch. Preis, et al., SPIE Proc. 3480, 236 (1998).

[11] L.N. Bulaevskii, et al., Europhys. Lett. 58, 415 (2002).

[12] D. van der Marel, et al., Phys. Rev. B 64, 024530 (2001).

[13] B. Gorshunov et al., to be published.

[14] S.N. Artemenko et al., JETP Lett. 58, 445 (1993); Physica C 253, 373 (1995).

[15] M. Norman, private communication.

[16] Ch. Helm, et al., submitted to Phys. Rev. B.

[17] S. Uchida, et al., Physica C, 293, 1 (1997).

[18] Y.I. Latyshev, et al., Phys. Rev. Lett. 825345 (1999).

[19] L. N. Bulaevskii, et al., JETP Lett 25, 290 (1977).

[20] L. Bartosch, et al., Phys. Rev. B 595377 (1999); W. Heindl, el al., J. Phys.: Cond. Matt. 12, 2245 (2002). 